\title{
Relación familia-escuela en el contexto de diversidad social y cultural, desde un enfoque educativo intercultural: principales tensiones epistemológicas
}

Zapata, Viviana Alejandra

Relación familia-escuela en el contexto de diversidad social y cultural, desde un enfoque educativo intercultural: principales tensiones epistemológicas

Revista Educación, vol. 46, núm. 1, 2022

Universidad de Costa Rica, Costa Rica

Disponible en: https://www.redalyc.org/articulo.oa?id=44068165001

DOI: https://doi.org/10.15517/revedu.v46i1.43800

\section{(c) $($ ) $\Theta \Theta$}

Esta obra está bajo una Licencia Creative Commons Atribución-NoComercial-SinDerivar 3.0 Internacional. 


\title{
Relación familia-escuela en el contexto de diversidad social y cultural, desde un enfoque educativo intercultural: principales tensiones epistemológicas
}

\author{
Family-School Relationships within a Context of Social and Cultural Diversity based on an Intercultural and \\ Educational Approach: Principal Epistemological Arguments
}

\author{
Viviana Alejandra Zapata \\ Universidad Católica de Temuco, Chile \\ viviana.alejandra.zapata@gmail.com \\ https://orcid.org/0000-0001-7367-9294
}

DOI: https://doi.org/10.15517/revedu.v46i1.43800

Redalyc: https://www.redalyc.org/articulo.oa? $\mathrm{id}=44068165001$

Recepción: 19 Octubre 2020

Aprobación: 20 Enero 2021

\section{RESUMEN:}

El objetivo de este ensayo es describir y analizar los principales factores que influyen en la relación familia-escuela, en contexto de diversidad social y cultural, desde la perspectiva étnico-cultural, específicamente, la población indígena y gitana. Esta descripción y análisis se realiza desde un enfoque educativo intercultural, que permite explicar las tensiones epistemológicas que se suscitan para establecer relaciones de colaboración entre la familia y la escuela. Lo anterior debido a los conocimientos y marcos explicativos distintos que tienen los agentes educativos y los agentes sociales que son parte de la escuela. El procedimiento de análisis de la información es el análisis de contenido. Los principales resultados se relacionan con: 1) la existencia de tensiones epistemológicas en la relación familia-escuela; 2) la participación como base para establecer una relación de colaboración entre la familia y la escuela; y 3) el enfoque educativo intercultural, que posibilita un proceso de co-construcción en la relación familia-escuela. Esto implica pistas de acción para personas directoras, profesoras, padres, madres y agentes sociales, que potencian una relación de colaboración entre agentes educativos y agentes sociales, esto desde la justicia epistémica. Se concluye que la relación familia-escuela en contexto de diversidad cultural, en espacios sociales determinados, se dinamiza a partir de la distancia y tensión epistemológica, lo que limita la relación de colaboración, a pesar de los esfuerzos políticos y educativos. Sin embargo, al implementar un enfoque educativo intercultural crítico como marco para la vigilancia epistémica, que potencia el reconocimiento de la alteridad y la relación entre agentes educativos y sociales. Por consiguiente, es la escuela, a partir del profesorado, la que debe planificar espacios dentro y fuera de la escuela, para co-construir una nueva forma de relación familia-escuela.

Palabras Clave: Tensiones, Familia-escuela, Colaboración, Enfoque educativo intercultural, Multiculturalidad, Justicia epistémica.

\section{ABstraCt:}

This study aims at describing and analyzing the principal characteristics of a family's relationship with their children's school. These aspects are considered within a context of social and cultural diversity and an ethnic-cultural perspective, specifically among indigenous and Roma population groups. Descriptions and analyses are based on an intercultural educational approach, which explain epistemological arguments used to establish collaborative relationships between the family and school, reflecting the diverse explanatory and knowledge-based frameworks of educational and social agents at the school.The main results are based on content analysis and consider: 1) epistemological tensions that characterize the family's relationship with their child's school; 2) participation as a basis for establishing a collaborative relationship between the family and the school; and 3) the intercultural educational approach which enables a process of co-construction in the family-school relationship. This requires a courses of action for managers, teachers, fathers, mothers and social agents for promoting a collaborative relationship among educational agents and social agents, from epistemic justice.

KeYwORDS: Tensions, Family-School, Collaboration, Intercultural Educational Approach, Multiculturalism, Epistemic Justice.

\section{INTRODUCCIÓN}

El presente ensayo aborda la relación familia-escuela en contexto de diversidad social y cultural, desde la perspectiva étnico-cultural, a partir del escenario migrante, como es el caso de la población gitana en 
España y el escenario indígena en Latinoamérica, específicamente en Chile (Bertely, 2016; Llevot y Bernad, 2016). En este entendido, se asume que la diversidad cultural es un fenómeno propio al ser humano y a la evolución que experimenta a lo largo de la historia, donde la otredad, que son parte de una determinada nación, debe enfrentarse desde distintas coyunturas o enfoques, por ejemplo: 1) el pluralismo cultural; 2) el multiculturalismo; y 3) la interculturalidad (Fajardo, 2014).

Desde esta perspectiva de evolución de los seres humanos y cambios en sus organizaciones, es que estas se ven desafiadas y tensionadas por el vertiginoso proceso de globalización, que ha posibilitado que muchas barreras sociales, políticas, económicas y culturales, que antes estaban cerradas, ahora se tengan que abrir (Mondaca y Gajardo, 2015). Esta situación ha generado distintas problemáticas, dentro de las que destacan la desigualdad, el racismo y la migración, lo que se traduce en nuevas conceptualizaciones y acciones que deben ser abordadas desde el contexto sociopolítico, para así responder a estas necesidades propias de procesos de transformación de la humanidad, que se evidencian a partir de otras formas de comprender el mundo desde la diversidad étnico-racial, que se comienzan a configurar en todos los espacios sociales (Fraser, 2008; Santos, 2016). Estas nuevas formas que los agentes sociales y políticos, dentro del contexto sociopolítico deben asumir, buscan ser coherentes con los proyectos Estado-Nación que cada territorio tiene (Quilaqueo y Quintriqueo, 2017). En el caso de España, con la alta migración gitana, se busca que esta sea parte del proyecto nacional, a partir del paradigma del multiculturalismo (Llevot y Bernad, 2016). En el caso indígena latinoamericano, se busca la incorporación de estas epistemes a partir del paradigma de la interculturalidad, que se inicia desde una perspectiva compensatoria y funcionalista; sin embargo, presenta el desafío de avanzar a una interculturalidad crítica (Williamson y Flores, 2015).

Esta relación entre la familia-escuela, respecto de la diversidad cultural, se lleva cabo a partir de la implementación de una interculturalidad funcional que limita la lectura emancipadora de la alteridad, que habilita la insurgencia de relaciones y otras subjetividades dentro y fuera de la sala de clases (Fraser, 2008; Madrid, Saracostti, Reininger y Hernández, 2019). Por lo tanto, es relevante describir y analizar la relación familia-escuela a partir de los procesos de colaboración que existen o no entre agentes educativos y agentes sociales, esto desde un enfoque educativo intercultural (Quilaqueo y Quintriqueo, 2017; Sartorello, 2016).

La relación familia-escuela se comprende como una construcción paradigmática y social, que articula subjetividades e intersubjetividades en el entramado relacional, tanto dentro como fuera de las instituciones educativas (Madrid et al., 2019). Esta relación que aparentemente es evidente, desde el punto de vista de la norma educativa, parece no ser tan concreta, desde el punto vista práctico en las acciones que se dinamizan a nivel escolar y familiar (Ortega y Cárcamo, 2018; Razeto, 2018). Esto se evidencia en los tipos de relación que se establece entre familia-escuela, que muchas veces operan de manera asimétrica y unidireccional por parte de la escuela. Esto implica que la institución formal opera desde marcos de referencia monoculturales, como son los conocimientos y normas escolares, a partir de un currículum y dispositivos prescritos (Matus, 2020; Perrenoud, 2010; Plá, 2016). Asimismo, en muchas oportunidades omiten o niegan los conocimientos propios de las familias y sus normas comportamentales, lo que genera una distancia epistemológica entre la escuela y la familia; en consecuencia, limita las relaciones colaborativas (Sartorello, 2016; Gasché, 2013).

La distancia epistemológica en la relación familia-escuela se intensifica en contextos de diversidad social y cultural desde la perspectiva étnico-racial, esto a pesar de los esfuerzos de la política educativa para fomentar prácticas democráticas y dialógicas, que explicitan como punto de partida el reconocimiento de la diversidad cultural, tanto para migrantes como indígenas (Jiménez y Fardella, 2015). Las acciones se orientan desde una mirada compensatoria, de déficit, o bien, desde la diferencia superficial, tal como señala Lepe-Garrión (2018), quien menciona que el multiculturalismo es una alternativa funcional a las políticas neoliberales, que, a partir de acciones de aceptación de diferencia, tipifican las diferencias culturales, las cuales se abordan desde un dominio y control hegemónico.

La educación escolar en contexto de colonización y migración tiene un carácter eminentemente civilizatorio, que se traduce en un sistema organizado, a nivel macro, meso y micro, desde una colonialidad 
del saber y del poder (Gasché, 2013; Quijano, 2000); esto implica una educación escolar que solo considera conocimientos occidentales y anula otros tipos de saberes al momento de diseñar, implementar y evaluar las políticas educativas (Plá, 2016). Por tanto, la escuela ha sido el vehículo que ha posibilitado la reproducción epistémica de un saber occidental y monocultural, arraigado en el colonialismo, que tiene como ideal instalar o fortalecer el proyecto de Estado-Nación (Foucault, 1992; Santos, 2016).

Es necesario tener en consideración que todo sistema educativo está orientado por paradigmas que subyacen a toda acción, lo que significa que no es inocente su toma de decisiones, organización y prácticas. Sobre todo, si con ello se fundamenta una episteme hegemónica eurocéntrica occidental (Gasché, 2013; Quilaqueo y Quintriqueo, 2017), que no siempre toma como punto de referencia la distinción social y cultural, como es el caso de las familias minorizadas o familias inmigrantes (Quilaqueo, 2019; Walsh, 2009). Dicha situación conlleva acciones reproductivas de castas, que niega los saberes propios de cada sociedad, que son reproducidas por la escuela, la educación escolar y los agentes que allí participan. Este tipo de acción puede ser de forma consciente o inconsciente, como el caso del profesorado, independiente del rol que tenga dentro de la escuela.

Las acciones reproductivas que se llevan a cabo en la escuela, en alguna medida, se explica con racismo biológico y epistémico, que se ha institucionalizado en todas las esferas sociales e incluso en las familias y grupos subalternizados, como es el caso de la población indígena (Quilaqueo, 2019). Por lo tanto, se explicita la ausencia de estrategias y comunicación dialógica por parte del profesorado, debido a la falta de preparación para escenarios diversos desde la formación inicial (Turra, Ferrada y Villena, 2013). Por consiguiente, queda limitada la capacidad para trabajar junto con las familias, para construir un bien común y contribuir a la justicia social desde la simetría de los conocimientos.

En ese contexto, se comprende que el rol del profesorado, estudiantado, padres, madres y agentes sociales se desarrolla en espacios con códigos culturales, ritos escolares y validación de conocimientos hegemónicos (Belavi y Murillo, 2020; Gramsci, 2013;). Sin embargo, estos pueden ser confrontados y transformados mediante la resistencia, la reflexión crítica y el lenguaje de posibilidades en los actores educativos y sociales (Giroux, 2011). Dicha transformación a partir del enfoque educativo intercultural, que asume la ecología de saberes como marco de referencia para la construcción de saberes multipolarizados y multirreferenciales (Bertely, 2016; Gasché, 2013; Santos, 2016).

A partir de estos elementos presentados, se establecen resistencias en las relaciones familia-escuela, las cuales se tensionan a nivel educativo y social, a pesar de las investigaciones y la evidencia del conocimiento acumulado (Epstein, 2018; Razeto, 2018). En este contexto, ambos agentes, familia y profesores, son complementarios y se necesitan mutuamente a partir de procesos de colaboración (UNESCO, 2017). Sin embargo, esta no es una acción permanente y transversal en el sistema educativo y más aún en familias minoritarias e inmigrantes (Llevot y Bernad, 2016).

Desde el enfoque educativo intercultural, existen investigaciones respecto a los procesos de colaboración entre familia-escuela, que permiten la relación entre conocimientos escolares y conocimientos racialesétnicos, que pretenden comprender a la educación como un espacio que capacita a estudiantes, profesores, profesoras, padres, madres y agentes sociales, en una conciencia de libertad para comprender la relación entre conocimiento y poder (Quijano, 2000; Sotero, Chaves, Gomes y Trindade, 2020).

En esta perspectiva se asume que, desde el enfoque educativo intercultural, es posible el estudio y la comprensión entre personas pertenecientes a sociedades y culturas diferentes, para construir un saber multipolarizado, con el fin de superar la discriminación, el racismo en la educación escolar y superar las tensiones epistemológicas que se dan dentro y fuera de la escuela en las relaciones entre familia-escuela (Quilaqueo y Quintriqueo, 2017; Turra et al., 2013). Por consiguiente, son el profesorado y los distintos agentes educativos quienes deben potenciar la construcción de distintas experiencias educativas orientadas desde la diversidad y los derechos humanos. 
El procedimiento de análisis de la información es el análisis de contenido para interpretar y develar nuevos antecedentes, que permitan discutir en relación con el objeto de estudio. El objetivo es describir y analizar los principales factores que influyen en la relación familia-escuela, en contexto de diversidad social y cultural, desde la perspectiva étnico-cultural, específicamente la población indígena y gitana.

\section{RELACIÓN FAMILIA-ESCUELA; UNA CONSTRUCCIÓN SOCIAL Y PARADIGMÁTICA}

La relación familia-escuela, desde el punto de vista de la política educativa, es una declaración de principios y acciones para implementar en las escuelas de forma democrática (Llevot y Bernad, 2016). Sin embargo, establecer una relación entre agentes educativos y agentes sociales presenta tensiones en cuanto al aspecto paradigmático, epistemológico y práctico, lo que muchas veces complejiza la relación entre la familia y la escuela a nivel consciente o inconsciente, y genera una distancia entre el profesorado, padres y madres. Por consiguiente, el estudiantado es quien asume los resultados de esta falta de relación (Etxebarria, Intxausti y Joaristi, 2013).

Diversos estudios indican que la relación familia-escuela se ve tensionada por distintos factores, dependiendo si es minoría étnica o si es inmigrante. En el primer caso se identifica una falta de participación debido a la carencia o déficit de conocimiento de orden escolar por parte de las familias indígenas (Bertely, 2016; Llevot y Bernad, 2016; Quilaqueo, 2019; Walsh, 2009). Respecto del segundo caso, se atribuye al desinterés de las familias por incorporarse a lógicas de funcionamiento distintas a sus patrones culturales (Cárdenas-Rodríguez, Terrón-Caro y Monreal, 2019; Río, 2010). Sin embargo, otros estudios (Ortega y Cárcamo, 2018; Madrid et al., 2019) interpelan el rol de la escuela frente a la tensión y distancias que se establecen con las familias, debido a que se sitúa desde un plano hegemónico, directivo y de transmisión de conocimiento por encima un plano dialógico y democrático, que fomente relaciones simétricas de poder entre el profesorado, estudiantado y sus familias. En este contexto, para Masschelein y Simons (2017):

El elemento democrático -y político- de la educación se ubica en esta doble experiencia del mundo como un bien común y 'puedo' (en oposición a 'debo'). Es la apertura de un mundo fuera de nosotros y la participación del niño o joven en este mundo compartido (p. 99).

En este sentido, la figura del profesorado tiene un rol relevante para establecer vínculos familia-escuela en contexto de diversidad social y cultural étnico-racial (Bertely, 2016); esto en relación con las expectativas que explicitan las familias respecto al rendimiento escolar y el comportamiento del estudiantado (Etxebarria et al., 2013; Santos, Lorenzo y Priegue, 2011). En consecuencia, las acciones que este grupo planifique e implemente tendrán distintos resultados dependiendo del enfoque al que estén supeditados, lo que suscita una problemática epistemológica y práctica (Ortega y Cárcamo, 2018). Esta situación se provoca debido a que el profesorado recibe una formación inicial que no siempre lo prepara para potenciar la relación familiaescuela, lo que se complejiza aún más en contextos de diversidad social y cultural (Turra et al., 2013). Por tanto, el profesorado no cuenta con todas las competencias desde su formación inicial para desarrollar un currículum intercultural.

Las personas docentes son parte de un sistema educativo escolar, donde sus creencias respecto al estudiantado funcionan como una profecía autocumplida (Turner y Chistensen, 2009), lo que muchas veces conlleva percepciones estereotipadas, reproducción de castas, culturas de bajas expectativas de acuerdo con ciertos parámetros sociales, culturales y económicos. En tal sentido es que, en sus prácticas, presentarán más y mejores oportunidades al estudiantado que atribuyeron mayores expectativas, tal como señala Bourdieu y Passeron (2001) sobre el sistema de creencia del profesorado y cómo las expectativas que generan en su accionar pedagógico están directamente relacionadas con el aprendizaje del estudiantado.

Respecto a la dimensión familiar, se ha evidenciado a partir de distintos estudios la baja participación familiar en la escuela (Cárdenas-Rodríguez et al., 2019; Etxebarria et al., 2013), lo que evidentemente 
limita que se establezca una relación entre ambas. Se entiende que la participación es el primer paso para potenciar una relación de colaboración entre ambos agentes (Ortega y Cárcamo, 2018), por consiguiente, si la participación es de baja intensidad, se complejiza avanzar hacia el vínculo más profundo, como la colaboración.

La relación familia-escuela se puede construir a partir de una dinámica dialógica, pues las personas se encuentran con más necesidades de comunicarse y dialogar, respecto de diferencias y similitudes que comparten o bien les diferencian (Flecha, 2018). Esto no implica que las relaciones de poder hayan desaparecido totalmente, pero sí ofrece una oportunidad al diálogo para visibilizar a los sujetos que eran invisibles y potenciar la resolución de conflictos explícitos e implícitos. Esto se evidencia desde las distintas ciencias del aprendizaje, donde el diálogo y sus componentes son relevados para la construcción de aprendizaje mediante la interacción, el diálogo, las relaciones horizontales y democráticas (Flecha, 2018; Olivé, 2009). En este contexto, es que a toda práctica educativa subyace una orientación dialógica, que conlleva mejores resultados de aprendizaje en el estudiantado, las significaciones sociales, culturales y la mejora de la convivencia.

Todas las intervenciones que se basen en una relación dialógica deben estar orientadas desde la participación de los agentes del medio educativo (personal directivo, profesorado y estudiantado) y social (padres, madres, sabios, dirigentes sociales, miembros de la comunidad), donde el diálogo y la valoración de la diversidad sea una práctica fundamental y dinamizadora de la acción educativa (Redecker y Punie, 2013). Esto potenciará a que cada agente educativo o social se sienta impulsado a ser más activo dentro del proceso educativo y a que el rol del profesorado les otorgue mayor seguridad y confianza (Rincón-Gallardo, 2016), al reconocer que existe en la escuela una pluralidad de epistemes.

\section{EL CASO GITANO E INDÍGENA DESDE EL INTERCULTURALISMO Y MULTICULTURALISMO}

Los conocimientos que están presentes en la sociedad, en la escuela y en la sala de clases son diversos, por lo tanto, es menester repensar las acciones pedagógicas y las formas de vincularse con la familia. El profesorado ha configurado esta relación desde un enfoque tradicional de educación, eminentemente centrado en un colonialismo arraigado, que complejiza avanzar a un proceso de articulación de conocimientos distintos que se pueden dar dentro y fuera de la escuela (Mampaey y Zanoni, 2015). En el entendido que el conocimiento es socialmente construido, como un proceso que asume que cada sociedad humana crea un cuerpo de conocimiento, a partir de múltiples experiencias o recursos en su vida diaria, las escuelas son espacios donde el estudiantado y el profesorado tienen la oportunidad de darse cuenta en la práctica de cómo la ciencia y otras formas de conocimiento pueden conectarse y beneficiarse mutuamente (Sotero et al., 2020).

En la última década, diversos estudios de educación intercultural han acuñado distintas perspectivas de esta; se destaca la de Bertely, Dietz y Díaz (2013), que explicitan dos grupos de personas investigadoras, tales como: 1) investigadores e investigadoras multiculturalistas, que buscan una apertura y aceptación de las diferencias sociales y culturales desde el diálogo, el respeto y la tolerancia en la escuela, que toma como referente una cultura globalizada; y 2) investigadoras e investigadores interculturalistas críticos, quienes orientan los procesos desde el enfoque descolonizador a partir de las asimetrías de poder imperante en las relaciones educativas.

Respecto al enfoque multiculturalista, este se ha utilizado principalmente en España para afrontar, desde el punto de vista educativo, los procesos de migración, como el caso de la población gitana, que lleva viviendo más de quinientos años en ese territorio (Cárdenas-Rodríguez et al., 2019). El multiculturalismo se asume desde un enfoque asimilacionista, en el que persiste la dominación de una cultura en relación a la otra y que en este caso particular ha transitado por cuatro etapas de implementación: 1) escuela solo para personas gitanas, con foco eminente de segregación cultural y social; 2) escuelas interétnicas, pero con aulas segregadas solo para personas gitanas; 3) escuelas en barrios que solo asisten personas gitanas; y 4) escuelas públicas 
diversas, a las que deben asistir las personas gitanas con estudiantes de otros orígenes étnico-raciales (Llevot y Bernad, 2016). Esta última etapa es la que contribuye a que las escuelas puente sean cerradas para avanzar a la integración de las personas gitanas a las aulas comunes. Sin embargo, esto genera resistencia de los agentes educativos y los agentes sociales, lo que complica la relación entre ambos.

Si bien existen tensiones entre las familias gitanas y profesionales que son parte de las escuelas, se pueden explicitar intentos de solución de la problemática, tales como: 1) la mediación gitana, que corresponde a una forma de vínculo entre la escuela y familia a partir de personas que son gitanas y que se instalan dentro instituciones educativas. Este tipo de práctica tiene una alta valorización de las familias, debido a que nace de ellos esta forma de vínculo (Llevot y Bernad, 2016); y 2) participación informal de las familias, ya que las relaciones informales con foco comunitario son altamente valoradas por las familias gitanas a la hora vincularse con la escuela, debido a que le otorgan un sentido más práctico (Cárdenas-Rodríguez et al., 2019).

En los últimos treinta años ha existido un intento de vinculación entre el currículum escolar y el patrimonio cultural de los pueblos indígenas en América Latina. Dicho intento no ha estado exento de dificultades de pertenencia histórica, debido a las tensiones epistemológicas, que subyacen a las comprensiones de cómo se construye el conocimiento y cuáles deberían ser los marcos curriculares que dinamicen las prácticas pedagógicas de la escuela (Aravena, 2017; Plá, 2016). En este sentido, no se puede olvidar que el currículum escolar tiene elementos de reproducción cultural (Birch, 2010) que se visibilizan en las acciones prácticas de la escuela. La modificación de este es una oportunidad de transformación de prácticas pedagógicas y cambios epistemológicos que potencien un vínculo familia-escuela (Birch, 2010; Wang, 2015).

En el caso de Chile, la educación intercultural tiene un marco normativo estandarizado y homogeneizante, cuyo paradigma propicia la negación social y cultural de los pueblos originarios (Aravena, 2017; Plá, 2016; Sartorello, 2016). Por consiguiente, se presenta con una visión monocultural, sustentada por un enfoque eurocéntrico occidental (Arias-Ortega, Quilaqueo y Quintriqueo, 2019). Así, el desafío de la política educativa, el currículum escolar y las prácticas pedagógicas es asumir una perspectiva intercultural pertinente y contextualizada a las distintas realidades socioculturales a las que pertenece el estudiantado (Bertely, 2016; Quintriqueo y McGinity, 2009). Por consiguiente, la formación de docentes en el contexto de la interculturalidad es un desafío, pues deben articularse los aspectos teóricos y prácticos desde los saberes sociales y culturales al abrir paso a las experiencias prácticas como propósito la superación de prácticas monoculturales y racistas (Giroux, 2011; Tubino, 2014; Quilaqueo y Quintriqueo, 2017).

En el contexto normativo nacional chileno existen políticas que buscan que los pueblos indígenas se involucren en la legislación y decisiones de orden educativa, tales como: 1) Ley Indígena $n^{\circ} 19253$, de 1993; y 2) Ley General de Educación n 20.370 (Williamson y Flores, 2015). Estas leyes han posibilitado la implementación de intentos de solución de la asimetría de conocimientos que se inician con las políticas orientadas a las personas indígenas y campesinas alrededor del año 1930, bajo una orientación indigenista que dinamizó las prácticas pedagógicas de las escuelas rurales a partir de los programas de educación indígena y el fortalecimiento de la educación rural (Williamson y Flores, 2015). Este proyecto permitió un avance significativo de las políticas compensatorias; sin embargo, se fundamenta en una perspectiva colonialista y monocultural (Williamson y Flores, 2015; Arias-Ortega, Quilaqueo et al., 2019), ya que el proceso legitima una racionalidad hegemónica sobre la racionalidad de grupos subalternizados (Nascimento, Quadros y Fialho, 2016). Dichos lineamientos normativos han tensionado la relación familia-escuela debido a los marcos explicativos del mundo que cada uno construye desde sus propias lógicas de conocimientos.

A partir de las experiencias presentadas, como es el caso gitano en España e indígena en Chile, y a partir de un enfoque multiculturalista e interculturalista funcional, es que la escuela debe establecer acciones emancipadoras, que permitan construir marcos de acción crítica (Giroux, 2011), no solo desde el reconocimiento de la diferencia, sino también desde la redistribución, que para este caso corresponde a la simetría del conocimiento en la relación familia-escuela (Fraser, 2008). Por lo que es congruente avanzar hacia un enfoque educativo intercultural crítico (Quilaqueo y Quintriqueo, 2017; Sartorello, 2016), que permita 
no olvidar que las sociedades cambian de manera vertiginosa y que esto genera incertidumbre e inestabilidad, desde una perspectiva valórica y contextual, lo que genera que las instituciones sigan funcionando, pero con poco sentido para las personas que lo conforman y por ende pierden el valor de representación de las personas (Bauman, 2009). Esta transformación del conocimiento debe darse desde un sentido local hacia uno global, pues cada sociedad y territorio puede resguardar y proteger el conocimiento y, principalmente, los saberes sociales y culturales (Essomba, 2008; Gasché, 2013).

\section{Desafíos De LA ESCUEla DESDE EL ENFOQUE EDUCATIVO INTERCULTURAL PARA LA TRANSFORMACIÓN DE LA RELACIÓN FAMILIA-ESCUELA}

El enfoque educativo intercultural es el estudio y la comprensión entre personas pertenecientes a sociedades y culturas diferentes, lo que implica construir un saber multipolarizado con el fin de superar la discriminación y el racismo en la educación escolar (Essomba, 2008; Quintriqueo y McGinity, 2009; Quilaqueo et al., 2017; Tubino, 2014). Este enfoque se concibe como una alternativa de reflexión crítica, que permite la relación de saberes y conocimientos de los pueblos indígenas con el conocimiento escolar, puesto que considera los procesos e interacciones que unen y definen a cada individuo y a los grupos, al enfatizar en el diálogo que surge entre estas partes (Sartorello, 2016; Brossard, 2019; Essomba, 2008). Lo anterior se grafica en las oportunidades en que el estudiantado pueda desarrollar capacidades de pensamiento crítico, lo que le permite generar una implicancia entre sujetos para construir un proyecto social común que involucre a las familias (Quilaqueo, 2019; Tubino, 2014). Así, se facilita la comprensión del mundo desde diferentes perspectivas, al considerar que este aprendizaje se da en un contexto social y cultural determinado, lo que permite la construcción social del conocimiento no de forma aislada, ni fragmentada.

El enfoque educativo intercultural, establece una configuración compleja, que va más allá de la aceptación de las diferencias para construir una mejor comprensión del mundo desde lógicas sociales y culturales (Bertely, 2016; Gasché, 2013). Por un lado, están los paradigmas que subyacen a las culturas étnicoraciales y la occidental (Fraser, 2008; Quilaqueo y Quintriqueo, 2017); este es un proyecto que está en construcción y que compromete complejidades que no son tan fáciles de resolver, pues conlleva un debate polisémico y controversial a la vez (Walsh, 2009; Torres y Friz, 2020; Sartorello, 2016). En consecuencia, la interculturalidad no es solo un debate pedagógico, sino también epistemológico, ético, político, social y cultural (Gasché, 2013; Sartorello, 2016; Quilaqueo y Quintriqueo, 2017). Por consiguiente, la negación, el desconocimiento y la falta de comprensión de las lógicas sociales y culturales indígenas generan una descontextualización de los distintos programas educativos, sociales y culturales (Ferrão, 2010), lo que lleva a un distanciamiento entre la familia y la escuela (Ortega y Cárcamo, 2018).

La interculturalidad desde el punto de vista educativo es una oportunidad de superación de la asimetría de poder y la negación de saberes sociales y culturales (Quintriqueo y Mcginity, 2009; Ferrão, 2010). Sin embargo, seguir con los modelos educativos monoculturales es simplificar a la humanidad a partir de una visión de mundo limitado, que no es propio de una segunda generación que comprende la interculturalidad como un proceso complejo (Sartorello, 2016).

Desde el punto de vista de la pedagogía, la interculturalidad es entendida como un principio, más allá de una disciplina, que tiene la potencia de construir y reconstruir junto con distintos actores sociales y culturales (Ferrão, 2010; Olivé, 2009; Arias-Ortega et al., 2019). Esto implica que la educación intercultural debe asumir seis principios orientadores, tales como: 1) la enseñanza y el aprendizaje que potencian la inserción en comunidades, a partir del desarrollo de habilidades y destrezas; 2) los significados, las habilidades y las destrezas, que generan que las personas se vinculen e interactúen con el medio social, cultural y natural; 3 ) las comunidades y personas, que pueden tener metas y conceptualizaciones con puntos de encuentro o bien diferencias sustanciales; 4) se aprende a partir de las intersubjetividades en un contexto social, donde subyace la individualidad; 5) el aprendizaje se potencia por la diferencia; y 6) la acción dialógica interconecta los 
saberes de distinto orden (saberes populares, saberes indígenas y el conocimiento escolar) que deben orientar la práctica pedagógica (Quilaqueo y Quintriqueo, 2017).

En este escenario es que la colaboración familia-escuela debe situarse desde la participación de las familias a partir del empoderamiento de éstas en distintos ámbitos, principalmente el educativo, lo que debe ser mediado intencionalmente por docentes y agentes educativos que forman parte de la escuela (Garbacz, Herman, Thompson y Reinke, 2017; Weist, Garbacz, Lane, y Kincaid, 2017). Esto involucra que deben co-construirse nuevos modelos de colaboración, que tenga como punto de partida el reconocimiento de diversos saberes sociales y culturales, donde el diálogo abierto y democrático oriente relaciones simétricas, con el propósito de superar el rol tradicional de la escuela que centra la participación de la familia en actividades planificadas desde la unidireccionalidad, tales como: apoyo en tareas escolares, reuniones de personas apoderadas, charlas o talleres para padres y madres (Madrid et al., 2019) y avanzar a procesos de colaboración activo que permita a cada agente familia-escuela proponer nuevas formas de relación.

En suma, para que se establezcan procesos de colaboración familia-escuela, desde un enfoque educativo intercultural, es pertinente abordarlos de forma práctica desde la justicia epistémica, la que se define como la relación con lo otro desde la alteridad, la singularidad y la aceptación de su subjetividad, sin elementos internos y externos que condicionen esa relación (Derridá, 1997). Lo anterior comprende reflexionar respecto a los prejuicios identitarios que se grafican en las distintas acciones interpretativas y hermenéuticas de la realidad con otras personas. Por tanto, la justicia epistémica tiene como punto de partida la colonización, no solo desde el punto de vista político, sino más bien desde las relaciones de dominación que naturalizan la subalternización desde lo cultural, social y étnico (Mandujano, 2017; Ogbu, 1992).

En consecuencia, la escuela debe asumir una ecología de saberes, como paradigma epistémico, que se configura desde una perspectiva constructivista con una eminentemente acción social y participativa de los distintos sujetos y sus saberes, así como desde la comprensión de la jerarquía y poderes que les subyacen (Mandujano, 2017). Por consiguiente, la ecología de saberes, como paradigma epistémico, busca en la interculturalidad, un espacio de vigilancia de la frontera de los saberes, que implica reconocer y comprender los otros saberes, a partir de los conocimientos propios (Mandujano, 2017; Santos, 2016).

\section{Conclusiones}

En la actualidad, la escuela es un espacio que limita la relación de colaboración entre el profesorado y las familias, principalmente aquellas que pertenecen a contexto de diversidad social y cultural, ya sean grupos minorizados o inmigrantes. Esta distancia en la relación puede ser explicada desde el punto de vista epistemológico, paradigmático, social y práctico; asimismo, pueden ser conscientes o inconsciente, ya que muchas veces se inicia con una baja participación de las familias en las actividades que la escuela planifica, básicamente porque los sentidos y finalidades de la educación no son los mismos.

En este contexto, de distanciamiento en la relación familia-escuela, es que las normativas internacionales y nacionales han buscado enfoques que posibiliten el acercamiento, más allá de las diferencias epistemológicas, sociales y culturales de los agentes educativos y sociales. Esto ha implicado, generar esfuerzos en la aplicación de modelos, como el multiculturalismo y el interculturalismo, para afrontar y dar respuesta práctica a los diversos supuestos explicativos que se encuentran dialógicamente en las escuelas. Sin embargo, estos enfoques no han sido suficiente para dar respuesta a la distancia epistemológica entre la familia-escuela, debido a las problemáticas que dinamizan la escolarización en la perspectiva monocultural.

En el marco de la educación escolar, el contexto de colonización ha sido empleado como un medio de dominación, minorización y subalternización de los indígenas a la colonialidad del poder y del saber eurocéntrico occidental. Respecto a los inmigrantes, la arbitrariedad cultural es un agente regulador en la relación establecida bajo las normas de las escuelas y no los parámetros culturales propios. Esto implica que la formación inicial no prepara al profesorado para potenciarla relación familia-escuela desde los contextos 
de diversidad social y cultural, lo que involucra que el personal docente presente falta de información y estrategias sobre las distintas culturas minoritarias o inmigrantes que cohabitan en la escuela, deficiente incluso para su identificación y unido a ciertos prejuicios sobre conductas que se gestan como consecuencia de lo anterior y la expectativas que afectan al rendimiento académico, sin tomar en consideración otros aspectos como déficit lingüístico, desvinculación familiar o deficiente participación familiar.

Sin embargo, existen experiencias de éxito a nivel internacional, las que orientadas desde un pluralismo epistemológico permiten establecer relaciones de colaboración entre la familia y la escuela, lo que potencia en ir más allá de la participación en actividades planificadas por la escuela, sino aportar desde su propia episteme en actividades que tributen al desarrollo integral de los estudiantes, las que evidencian resultados de orden cualitativo y cuantitativo, debido a que se reconoce que cuando las madres y padres se comprometen con actitudes positivas hacia la escuela sus hijos e hijas fortalecen los resultados académicos y de convivencia escolar.

Bajo esta premisa de resistencia y distancia es que la comunicación que el profesorado mantenga con las familias de grupos minorizados y familias inmigrantes permitirá liberarlas de incertidumbres e inseguridad en ambos lados, debido a que la realidad de cada contexto ya sea el escolar o el familiar, muchas veces es desconocido por los distintos agentes. Es así, que la figura del profesorado es clave para generar acercamiento en la relación familia-escuela, lo que implica que la escuela debe repensar y resituar su rol. Por lo tanto, en pleno siglo XXI el desafío para la escuela es avanzar a una cultura de colaboración entre la familia-escuela, con el propósito de fortalecer la relación entre docentes, madres, padres y otros agentes de la familia a partir de un pluralismo epistemológico orientado hacia una práctica intercultural para la transformación de la escuela, para tributar a una mayor justicia social.

En suma, es necesario que las escuelas, a partir de agentes educativos como el profesorado, establezcan acciones desde la justicia epistémica a partir del reconocimiento de diferencia social y cultural, para implementar medidas que permitan una ecología de saberes. Para que esto ocurra es fundamental planificar instancias de reflexión con la familia, dentro y fuera de la escuela, para que de manera colectiva y dialógica se planifiquen rutas y actividades que posibiliten el diálogo de saberes diversos, que resignifiquen los sentidos de la escuela en el vínculo de colaboración familia-escuela para el siglo XXI.

\section{ReFerencias Bibliográficas}

Aravena, F. (2017). Análisis comparado sobre patrimonio cultural indígena y currículo: Australia, Chile y Sudáfrica. Calidad en la educación, (46), (pp. 165-192). doi: http://dx.doi.org/10.4067/S0718-45652017000100165

Arias-Ortega, K., Quilaqueo, D. y Quintriqueo, S. (2019). Educación intercultural bilingüe en La Araucanía: principales limitaciones epistemológicas. Educação e Pesquisa, (45), (pp. 1-16). doi: https://doi.org/10.1590/s $1678-4634201945192645$

Bauman, Z. (2009). En busca de la política. Buenos Aires, Argentina: Fondo de Cultura Económica.

Belavi, G. y Murillo, J. (2020). Democracia y Justicia Social en las Escuelas: Dimensiones para Pensar y Mejorar la Práctica Educativa. Revista Iberoamericana sobre Calidad, Eficacia y Cambio en Educación, 18(3), (pp. 5-28). doi: https://doi.org/10.15366/reice2020.18.3.001

Birch, E. (2010). Indigenous perspectives in the Australian curriculum. TLN Journal 17(2), (pp.16-18). Recuperado de https://bit.ly/3hcANSj

Brossard, L. (2019). Les peuples autochtones: des réalités méconnues à tout point de vue. La publication en ligne de I'ICÉA. (p. 76). Recuperado de https://bit.ly/3heJcoo

Bertely, M. (2016). Políticas neoliberales y afectaciones territoriales en México. Algunos "Para qués" de "otras" educaciones. Revista Liminar. Estudios Sociales y Humanisticos, 14(1), (pp. 30-46). Recuperado de: https://ww w.redalyc.org/articulo.oa?id=74543269003

Bertely, M., Dietz, G. y Díaz, M. (2013). Multiculturalismo y educación. México: COMIE, ANUIES. 
Bourdieu, P. y Passeron, J. (2001). La reproducción: Elementos para una teoría del sistema de enseñanza. Barcelona, España: Fontorama.

Cárdenas-Rodríguez, R., Terrón-Caro, T. y Monreal, M.C. (2019). Educación Primaria y alumnas gitanas. Análisis de las barreras sociales en contextos de exclusión. Revista de Investigación Educativa, 37(1), (pp. 75-91). doi: http ://dx.doi.org/10.6018/rie.37.1.326221

Derridá, J. (1997). Resistencias. En Resistencia del psicoanálisis. Buenos Aires, Argentina. Paidós.

Epstein, J. (2018). School, family, and community partnerships: preparing educators and improving schools. New York: Routledge. doi: https://doi.org/10.4324/9780429494673

Essomba, M. (2008). 10 ideas clave: La gestión de la diversidad cultural en la escuela. Barcelona, España: Graó.

Etxebarria, F., Intxausti, N. y Joaristi, L. (2013). Factores que favorecen la implicación educativa de las familias inmigrantes con hijos en Educación Primaria. Revista Psicodidáctica, 18(1), (pp. 109-136). doi: https://doi.org /10.1387/RevPsicodidact.5684

Fajardo, D. (2014). La diversidad cultural y las políticas culturales: la distancia entre la intolerancia y la integración. Paradigma: Revista De Investigación Educativa, 20(32), (pp.23-40). doi: https://doi.org/10.5377/paradigma.v $20 \mathrm{i} 32.1413$

Ferrão, M. (2010). Educación intercultural en América Latina: Distintas concepciones y tensiones actuales. Revista Estudios Pedagógicos, 36(2), (pp. 333-342). Recuperado de https://scielo.conicyt.cl/pdf/estped/v36n2/art19.p $\mathrm{df}$

Flecha, R. (2018). Comunidades de Aprendizaje y transformación social. Educadores: Revista de renovación pedagógica, (265), (pp. 44-54). Recuperado de https://dialnet.unirioja.es/ejemplar/485790

Fraser, N. (2008). Escalas de Justicia. Barcelona, España: Herder.

Foucault, M. (1992). Las redes del poder. Buenos Aires, Argentina: Almagesto.

Gasché, J. (2013). Éxitos y fracasos de una propuesta educativa basada sobre el "Método Inductivo Intercultural" e implementada en el Perú, México y el Brasil. Revista ISEES, (13), (pp. 17-31). Recuperado de https://bit.ly/3 bf $112 \mathrm{u}$

Giroux, H. (2011). On critical pedagogy. New York, NY: Bloomsbury.

Garbacz, S., Herman, K., Thompson, A. y Reinke, W. (2017). Family engagement in education and intervention: Implementation and evaluation to maximize family, school, and student outcomes. Journal of school psychology, 62, (pp. 1-10). doi:https://doi.org/10.1016/j.jpp.2017.04.002

Gramsci, A. (2013). Cuadernos de la cárcel. Los intelectuales y la organización de la cultura. México: Juan Pablós.

Jiménez, F. y Fardella, C. (2015). Diversidad y rol de la escuela: discursos del profesorado en contextos educativos multiculturales en clave migratoria. Revista Mexicana de Investigación Educativa, RMIE, 20(65), (pp. 419-441). Recuperado de http://www.scielo.org.mx/pdf/rmie/v20n65/v20n65a5.pdf

Lepe-Garrión, P. (2018). El discurso intercultural como campo de disputa: 'terrorismo mapuche' y dispositivo pedagógico de etnicidad. Revista Historia y justicia, (15), (pp. 311-347). Recuperado de: http://revista.historiayjusticia.org/varia/el-discurso-intercultural-como-campo-de-disputa-terrorismo-map uche-y-dispositivo-pedagogico-de-etnicidad/

Llevot, N. y Bernad, O. (2016). La mediación gitana: herramienta performativa de las relaciones entre escuela y familia. Revista Electrónica Interuniversitaria de Formación del Profesorado, 19(1), (pp. 99-110). Recuperado de: https:/ /revistas.um.es/reifop/article/view/245581/189001

Madrid, R., Saracostti, M., Reininger, T. y Hernández, M.T. (2019). Responsabilización, obediencia y resistencia: perspectivas de docentes y padres sobre la colaboración familia escuela. Revista Electrónica Interuniversitaria de Formación del Profesorado, 22(3), (pp. 1-13). doi: https://doi.org/10.6018/reifop.389801

Mandujano, M. (2017). Justicia epistémica y epistemologías del sur. Oximora revista internacional de ética y politica, (10), (pp. 148-164). Recuperado de https://raco.cat/index.php/Oximora/article/view/323223 
Mampaey, J. y Zanoni, P. (2015). Reproducing monocultural education: ethnic majority staff's discursive constructions of monocultural school practices. British Journal of Sociology of Education, 37(7), (pp. 1-19). Recuperado de http://www.tandfonline.com/doi/abs/10.1080/01425692.2014.1001059

Masschelein, J. y Simons, M. (2017). Em defesa da escola: uma questão pública. Tradução Cristina Antunes. (2. ed.). Belo Horizonte, Brasil: Autêntica Editora.

Matus, M. (2020). De Prácticas y discursos Universidad Nacional del Nordeste Centro de Estudios Sociales. Dossier: educación y pueblos indigenas, 13(9), (pp. 1-20). doi: http://dx.doi.org/10.30972/dpd.9134313

Mondaca, C. y Gajardo, Y. (2015). Interculturalidad, migrantes y educación. Diálogo andino, (47), (pp. 3-6). doi: ht tps://dx.doi.org/10.4067/S0719-26812015000200001

Nascimento, R., Quadros, M. y Fialho, V. (2016). Interculturalidade enquanto prática na educação escolar indígena. Revista Anthropológicas, 27(1), (pp. 187-217). Recuperado de https://periodicos.ufpe.br/revistas/revistaanthro pologicas/article/viewFile/24041/19503

Ogbu, J. (1992). Understanding Cultural Diversity and Learning. Educational Researcher, 21(8), (pp. 5-14). doi: htt p://dx.doi.org/10.2307/1176697

Olivé, L. (2009). Por una auténtica interculturalidad basada en el reconocimiento de la pluralidad epistemológica. Pluralismo epistemológico. La Paz: Muela del Diablo.

Ortega, M. y Cárcamo, H. (2018). Relación familia-escuela en el contexto rural. Miradas desde las familias. Educación, 27(52), (pp. 98-118). doi: http://dx.doi.org/10.18800/educacion.201801.006

Perrenoud, P. (2010). Desarrollar la práctica reflexiva en el oficio de enseñar. Barcelona, España: Graó.

Plá, S. (2016). Currículo, historia y justicia social. Estudio comparativo en América Latina. Revista Colombiana de Educación, (71), (pp.53-77). Recuperado de http://www.scielo.org.co/pdf/rcde/n71/n71a03.pdf

Quijano, A. (2000).Colonialidad del poder, eurocentrismo y América Latina. Dans Lander (dir.), Colonialidad del saber y eurocentrismo. Buenos Aires, Argentina: UNESCO-CLACSO.

Quilaqueo, D. (2019). Intervención educativa intercultural para un diálogo de saberes indígena y escolar. Educar em Revista, 35(76), (pp. 219-237). doi: http://dx.doi.org/10.1590/0104-4060.63000

Quilaqueo, D. y Quintriqueo, S. (2017). Métodos Educativos Mapuches: Retos de la doble racionalidad educativa, aportes para un enfoque educativo intercultural. Universidad Católica de Temuco, Chile.

Quintriqueo, S. y McGinity, M. (2009). Implicancias de un modelo monocultural curricular en la construcción de la identidad sociocultural de alumnos/as mapuches de la IX región de la Araucanía, Chile. Estudios Pedagógicos, 35(2), (pp.173-188). Recuperado de http://www.redalyc.org/articulo.oa?id=173514137010

Razeto, A. (2018). Estrategias para promover la participación de los padres en la educación de sus hijos: el potencial de la visita domiciliaria. Estudios Pedagógicos, 42(2), (pp. 449-462). doi: https://doi.org/10.4067/S0718-0705 2016000200026

Redecker, C. y Punie, Y. (2013). The Future of Learning 2025: Developing a vision for change. Future Learning, 2(1), (pp. 3-17). doi: https://doi.org/10.7564/13-FULE12

Rincón-Gallardo, S. (2016). Large-Scale Pedagogical Transformation as Widespread Cultural Change in Mexican Public Schools. Journal of Educational Change, 17(4), (pp. 411-436). doi: https://doi.org/10.1007/s10833-01 6-9286-4

Río, M. (2010). No quieren, no saben, no pueden: categorizaciones sobre las familias más alejadas de la norma escolar. Revista Española De Sociología, (14), (pp. 85-105). Recuperado de https://recyt.fecyt.es/index.php/res/article/ view/65180

Torres, H. y Friz, M. (2020). Elementos críticos de la escuela en territorio mapuche. Educar em Revista, Curitiba, (36), (pp. 66-108). doi: http://dx.doi.org/10.1590/0104-4060.66108

Tubino, F. (2014). El trasfondo episte\#mico de los conflictos interculturales. Revista Contextualizaciones Latinoamericanas, 6(11), (pp. 1-5). Recuperado de https://core.ac.uk/download/pdf/322549178.pdf

Turner, J.y Christensen, A. (2009). Collaborating with teachers to foster student motivation: Implementing motivational strategies in mathematics instruction. American Educational Research Association, Chicago. 
Turra, O., Ferrada, D. y Villena, A. (2013). La especificidad del contexto indígena como requerimiento para la formación inicial del profesorado. Estudios Pedagógicos, 39(2), (pp. 329-339). doi: http://dx.doi.org/10.4067/ S0718-07052013000200020

Santos, M., Lorenzo, M. y Priegue, D. (2011). Infancia de la inmigración y educación: La visión de las familias. Revista de Investigación Educativa, 29(1), (pp. 97-110). Recuperado de https://revistas.um.es/rie/article/view/110351

Sartorello, S. (2016). Política, epistemología y pedagogía: el Método Inductivo Intercultural en una escuela tseltal de Chiapas, México. Revista LiminaR Estudios Sociales y Humanisticos, 14(1), (pp. 121-143). Recuperado de: http s://www.redalyc.org/pdf/745/74543269009.pdf

Santos, B. (2016). La difícil democracia. Una mirada desde la periferia europea. Madrid, España: Akal.

Sotero, M., Chaves, A., Gomes, A. y Trindade, M. (2020) Local and scientific knowledge in the school context: characterization and content of published works. Journal of Ethnobiology and Ethnomedicine, 16(23), (pp. 2-23). doi: https://doi.org/10.1186/s13002-020-00373-5

UNESCO. (2017). Conocimiento Indigena y Politicas Educativas en América Latina. Organización de las Naciones Unidas para la Educación, la Ciencia y la Cultura. Santiago de Chile, Chile: OREALC/UNESCO.

Walsh, C. (2009). Interculturalidad, Estado, Sociedad: Luchas decoloniales de nuestra época. Quito, Ecuador: Universidad Andina Simón Bolívar; Abya Yala.

Wang, C. (2015). The review of multicultural curriculum transformation on indigenous ethnic curriculum policy development. Journal of Curriculum Studies, 10(1), (pp. 13-34). doi: https://doi.org/10.3966/181653382015 031001002

Weist, M., Garbacz, S. A., Lane, K. y Kincaid, D. (2017). Aligning and integrating family engagement in positive behavioral interventions and supports (PBIS): Concepts and strategies for families and schools in key contexts. Center for Positive Behavioral Interventions and Supports (funded by the Office of Special Education Programs, US Department of Education). Eugene, OR: University of Oregon.

Williamson, G. y Flores, F. (2015). Estado Del Arte De La Educación Intercultural Bilingüe En Chile, 1990-2013. Temuco, Chile: Ediciones Universidad de la Frontera Facultad de Educación, Ciencias Sociales y Humanidades.

\section{INFORMACIÓN ADICIONAL}

Cómo citar: Zapata, V. A. (2022). Relación familia-escuela en el contexto de diversidad social y cultural, desde un enfoque educativo intercultural: principales tensiones epistemológicas. Revista Educación, 46(1). http:// doi.org/10.15517/revedu.v46i1.43800 